\title{
GENERALIZED REGRESSION NEURAL NETWORK BASED EXPERT SYSTEM FOR HEPATITIS B DIAGNOSIS
}

\author{
${ }^{1}$ Mahesh, C., ${ }^{2}$ E. Kannan and ${ }^{1}$ M.S. Saravanan \\ ${ }^{1}$ Department of IT, \\ ${ }^{2}$ Department of CSE, \\ Vel Tech University, Avadi, Chennai, India
}

Received 2013-08-06; Revised 2013-08-31; Accepted 2013-12-03

\begin{abstract}
Hepatitis B is a potentially life-threatening liver infection caused by the hepatitis B virus. The virus interferes with the function of the liver while replicating in hepatocytes. It is a major global health problem and the most serious type of viral hepatitis. Chronic liver disease is caused by viral hepatitis and putting people at high risk of death from cirrhosis of the liver and liver cancer. Medical information available is extensive and which is utilized by the clinical specialists. The ranging of information is from details of clinical symptoms to various types of biochemical data. Information provided by each data is evaluated and assigned to a particular pathology during the diagnostic process. Artificial intelligence methods especially computer aided diagnosis and artificial neural networks can be employed to streamline the diagnostic process. These adaptive learning algorithms can handle diverse types of medical data and integrate them into categorized outputs. Artificial neural networks are finding many uses in the medical diagnosis application. In this study we have proposed a Generalized Regression Neural Network (GRNN) based expert system for the diagnosis of the hepatitis $\mathrm{B}$ virus disease. The system classifies each patient into infected and non-infected. If infected then how severe it is in terms of intensity rate.
\end{abstract}

Keywords: Expert System, Artificial Neural Networks, Generalized Regression Neural Network (GRNN), Hepatitis B Virus (HBV), Hepatitis B Nomenclature, Hepatitis B Diagnosis

\section{INTRODUCTION}

Since the Conventional manual data analysis techniques are not effective in diagnosis, using computer based analyses are becoming inevitable in disease diagnosis. So, it is the time to develop modern, effective and efficient computer based systems for decision support. There are a number of data analysis techniques: statistical, machine learning, expert system and data abstraction (Cheung, 2003). Recent practice for medical treatment make it mandatory that patients consult specialists for further diagnosis and treatment. Other medical practitioners may not have adequate expertise or experience in handling certain high-risk diseases. Nonetheless, typical waiting time for treatments may be few days, weeks or even months. Possibly, by the time the patients consult the specialists the diseases may have already spread out. Since the majority of the high-risk disease could only be cured at the early stage, the patients may have to endure for the rest of their life, due to which new approaches with the support of computer technology for the diagnosis of diseases is essential. The mortality rate and the waiting time to see the specialist could be reduced by employing the computer technology or computer program or software developed by emulating human intelligence which supports the doctors in making decisions without the direct consultation with the specialists.

Hepatitis B is caused by a virus that attacks the liver. This disease ranked as the third infectious one. The Corresponding Author: Mahesh, C., Department of IT, Vel Tech University, Avadi, Chennai, India 
importance of surveying and designing an intelligence system has been sensed in this field. A comparative Analysis of all neural networks proved that generalized regression neural network will be the best suitable network in diagnosis of Hepatitis B. The capability of Generalized Regression Neural Network to get trained faster compared to other networks and achieving results for even the few missing attribute makes it used for diagnosis process (Ozyilmaz and Yildirim, 2003). GRNN is a very useful tool to perform predictions and comparisons of system performance in practice.

\section{HEPATITIS B OVERVIEW}

HBV is classified in the family Hepadnaviridae. It occurs as seven distinct genotypes, designated $A$ to $G$, but it is controversial as to whether the outcome of the infection is influenced by the genotype (Stuyver et al., 2000). HBV has a double-stranded DNA genome of approximately 3200 base pairs organized into four partially overlapping open reading frames, which encode the envelope, core (precore/core), polymerase and $\mathrm{X}$ proteins. The envelope proteins are surface glycoprotein collectively designated as Hepatitis B surface Antigen (HBsAg). In virus-infected liver cells, HBsAg is excessively produce in virus infected liver cells and it is secreted into the blood, where it serves as a marker for active infection and infectivity. Recombinant HBsAg is used for $\mathrm{HBV}$ vaccination currently and the antibody development to HBsAg is associated typically with protective immunity.

\subsection{Data}

Applied data in this research for diagnosing the rate of $\mathrm{HB}$ intensity have been chosen from the patients. There are 300 records and each record has 8 fields. H_B (Intension Diagnosis hepatic B) is the $8^{\text {th }}$ field. This field is the specialist diagnosis that divides HB intensity levels into 4 parts:

- Normal situation (virus has not hurt vital organs yet)

- Light situation (virus has just started its destroying activity)

- Severe situation (some parts of vital organs have been destroyed)

- Hyper-severe situation (necessarily, patient needs intensive cares and even patient's life is in danger)

The above situations have been put into a table (Table 1) according to the specialists diagnosis in order to compare the resulting together after designing the expert system and the convention system.

\subsection{Recommended Tests to Investigate Chronic HBV Infection and the Interpretation of Results}

Chronic HBV infection is defined by the continued presence of HBsAg in the blood for longer than six months. Table 2 outline the tests used to diagnose most cases of chronic HBV. Test selection should be based on the person's risk factors, vaccination history and findings from previous tests.

Table 3 gives the usual interpretation for sets of results from Hepatitis B blood (serological) tests.

\section{ARTIFICIAL NEURAL NETWORKS}

The basic principle of neural network can be known from (Brause, 2001). Human neural architecture is represented in mathematical form is called Artificial Neural Network. ANN reflects the human neural abilities of learning and generalization. This is what the reason that ANNs belong to the field of artificial intelligence. In research Artificial Neural Networks are widely applied because highly non-linear systems can be modeled in which the relationship among the variables is unknown. A review of various classes of neural networks can be found in (Aleksander and Morton, 1995; Zupan and Gasteiger, 1999). Overview of the main applications of artificial neural networks in medicine is shown in the following Fig. 1.

\subsection{Mathematical Background}

Series of neurons form a neural network. The neurons are organized in layers. A neuron in one layer is connected with the neuron in the next layer through a weighted connection. The strength of the connection between neurons is indicated by weight value $\mathrm{w}_{\mathrm{ij}}$.

The input layer, one or more hidden layers and the output layer form the neural network structure. The complexity of the system decides the number of neurons in each layer and the number of layers. Therefore, the optimal network architecture must be determined. Typical three-layered ANN architecture of general scheme is given in Fig. 2.

Table 1. Different Situation output

\begin{tabular}{lll}
\hline S.no & State & Range \\
\hline 1 & Normal & $0-3$ \\
2 & Light & $3-5$ \\
3 & High & $5-7$ \\
4 & Very high & $7-10$ \\
\hline
\end{tabular}


Table 2. Primary and Secondary tests to diagnose/monitor hepatitis B virus (HBV) infection

\begin{tabular}{lllllll}
\hline & Marker & $\begin{array}{l}\text { Incubation } \\
\text { period }\end{array}$ & $\begin{array}{l}\text { Acute } \\
\text { infection }\end{array}$ & $\begin{array}{l}\text { Past/resolved } \\
\text { infection }\end{array}$ & $\begin{array}{l}\text { Chronic } \\
\text { infection }\end{array}$ & Vaccination \\
\hline Primary diagnostic tests & HbsAg & \pm & + & - & + & $-^{*}$ \\
& Anti-HBs & - & - & + & + & + \\
& Anti-HBc-Total & - & + & + & + & - \\
\multirow{5}{*}{ Prognostic or monitoring tests } & Anti-HBc-IgM & - & + & - & $\pm^{\dagger}$ & - \\
& HbeAg & \pm & + & - & \pm & - \\
& Anti-Hbe & - & - & \pm & $\pm^{\ddagger}$ & - \\
& HBV-DNA & $\pm^{\S}$ & + & $\pm^{\S}$ & $+^{\S}$ & - \\
\hline
\end{tabular}

*Recent HBV vaccination within one to two weeks can lead to a false-positive test. The vaccine antigen can be detected at low levels; $†$ May be positive in chronically infected individuals; $¥$ Patients with chronic HBV infection usually have detectable Hepatitis $\mathrm{B}$ e antigen ( $\mathrm{HBeAg}$ ) or antibody to hepatitis B e protein (anti-HBe). Rarely, both $\mathrm{HBeAg}$ and anti-HBe can be detected simultaneously; § Methods differ in sensitivity and standardization. Anti-HBc Antibody to hepatitis B core protein; Anti-HBs Antibody to hepatitis B surface protein; HBsAg Hepatitis B surface antigen; IgM Immunoglobulin M; + Implies positive; - Implies negative; \pm May be positive or negative

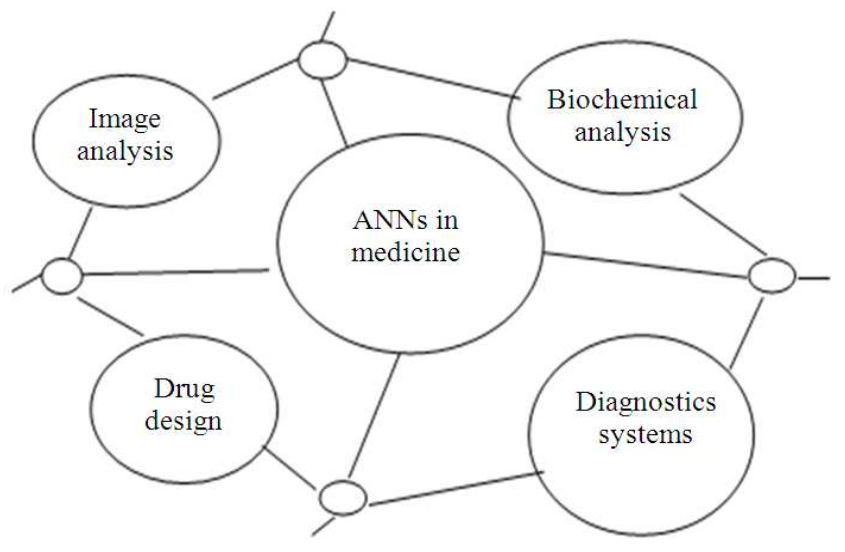

Fig. 1. Artificial neural networks applications in medicine

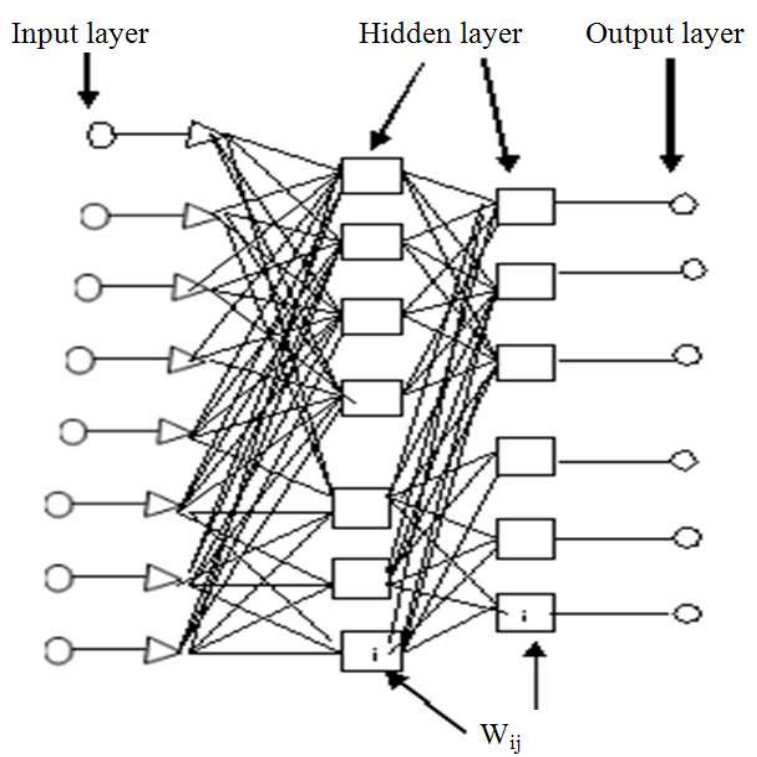

Fig. 2. Neural network structure with two hidden layers
Table 3. Markers for Hepatitis B diagnosis

\begin{tabular}{ll}
\hline Marker & Value \\
\hline HbsAg & Positive \\
HbsAg & Negative \\
HbeAg & Positive \\
HbeAg & Negative \\
Anti-VHD & Positive \\
Anti-VHD & Negative \\
Anti-VHC & Positive \\
\hline
\end{tabular}

Rule: If (HbsAg = Positive) and (HbeAg = Positive) and (anti$\mathrm{VHD}=$ Negative) then Hepatitis B

The data is received by the neurons in the input layer and transferred to first hidden layer neurons through the weighted links. The mathematically processed data and the result is transferred to the next layer neurons. Eventually the network's output is produced by the last layer neuron. The hidden layer k-th neuron processes the incoming data $\mathrm{Z}_{\mathrm{i}}$ by:

- Calculating the weighted sum and adding a "bias" term $\left(\theta_{\mathrm{k}}\right)$ according to Equation (1): 


$$
\begin{aligned}
& \text { net }_{\mathrm{k}}=\sum_{\mathrm{i}=1}^{\mathrm{m}}\left(\mathrm{z}_{\mathrm{i}} \times \mathrm{w}_{\mathrm{ik}}+\theta_{\mathrm{k}}\right) \\
& \text { where }(\mathrm{k}=1,2,3, \ldots, \mathrm{n})
\end{aligned}
$$

- Transforming the net $\mathrm{k}_{\mathrm{k}}$ through a suitable mathematical "transfer function"

- Result transferred to neurons in the next layer

Various transfer functions are available (MNI, 1996). However the sigmoid one which is most commonly used is Equation (2):

$$
f(z)=\frac{1}{1+e^{-z}}
$$

\subsection{Neural Network Learning}

The network achieves "learning" through the mathematical process can be ignored by the final user principally. This is the way of viewing the network as "black box". The black box receives a vector with " $n$ " inputs and provides a vector with "m" outputs (Fig. 3).

The network learns from a series of examples that form the training database (Fig. 4). An example is formed by a vector $\mathrm{X}_{\mathrm{im}}=\left(\mathrm{x}_{\mathrm{i} 1}, \mathrm{x}_{\mathrm{i} 2}, \ldots, \mathrm{x}_{\mathrm{im}}\right)$ of inputs and $\mathrm{a}$ vector $Y_{\text {in }}=\left(y_{i 1}, y_{i 2}, \ldots . ., y_{\text {in }}\right)$ of outputs. The objective of the training process is to approximate the function $f$ between the vectors $X_{\mathrm{im}}$ and the $\mathrm{Y}_{\mathrm{in}}$ :

\section{GENERALIZED REGRESSION NEURAL NETWORK (GRNN)}

The system modelling and prediction (MNI, 1996) proposed originally the generalized regression neural network. As the back-propagation network GRNN has been used to learn the same problems, the radial basis function network, the probabilistic neural network and the modular neural network. The generalized neural network has a relationship to the probabilistic neural network and mostly been used in place of it for classification problems.

This network has certain characteristics:

- Fast learning

- Good convergence with a large number of training examples

- Handling of sparse data well

- Possible memory hog

- Possible computing time issues
Generalized regression neural network belongs to the category of probabilistic neural networks. Like other probabilistic neural networks GRNN needs only a fraction of the training samples a back propagation neural network would need. Available data from measurements of an operating system is generally never enough for a back propagation neural network. Therefore the use of a probabilistic neural network is especially advantageous due to its ability to converge to the underlying function of the data with only few training samples available. The knowledge needed additionally to get the fit in a satisfying way is relatively small and can be done without additional input by the user. This will make GRNN a very useful tool to perform predictions and comparisons of system performance in practice.

\subsection{Architecture}

As it can be seen from Fig. 5, the Generalized Regression Network consists of three layers of nodes with entirely different roles:

- The input layer, where the inputs are applied

- The hidden layer, where a nonlinear transformation is applied on the data from the input space to the hidden space; in most applications the hidden space is of high dimensionality

- The linear output layer, where the outputs are produced

\subsection{Algorithm}

The probability density function used in GRNN is the Normal Distribution. Each training sample, $\mathrm{Xj}$, is used as the mean of a Normal Distribution:

$$
\begin{array}{r}
Y(X)=\frac{\sum_{i=1}^{n} Y_{i} \exp \left(-D_{i}^{2} / 2 \sigma^{2}\right)}{\sum_{i=1}^{n} \exp \left(-D_{i}^{2} / 2 \sigma^{2}\right)} \\
D_{i}^{2}=\left(X-X_{i}\right)^{T} \cdot\left(X-X_{i}\right)
\end{array}
$$

The distance, $D_{j}$, between the training sample and the point of prediction, is used as a measure of how well the each training sample can represent the position of prediction, X. If the Distance, $D_{j}$, between the training sample and the point of prediction is small, exp $\left(-D_{j}{ }^{2} / \sigma^{2}\right)$, becomes big. For $D_{j}=0$, exp ($\mathrm{D}_{\mathrm{j}}^{2} / 2 \sigma^{2}$ ) becomes one and the point of evaluation is represented best by this training sample. 
Mahesh, C. et al. / Journal of Computer Science 10 (4): 563-569, 2014

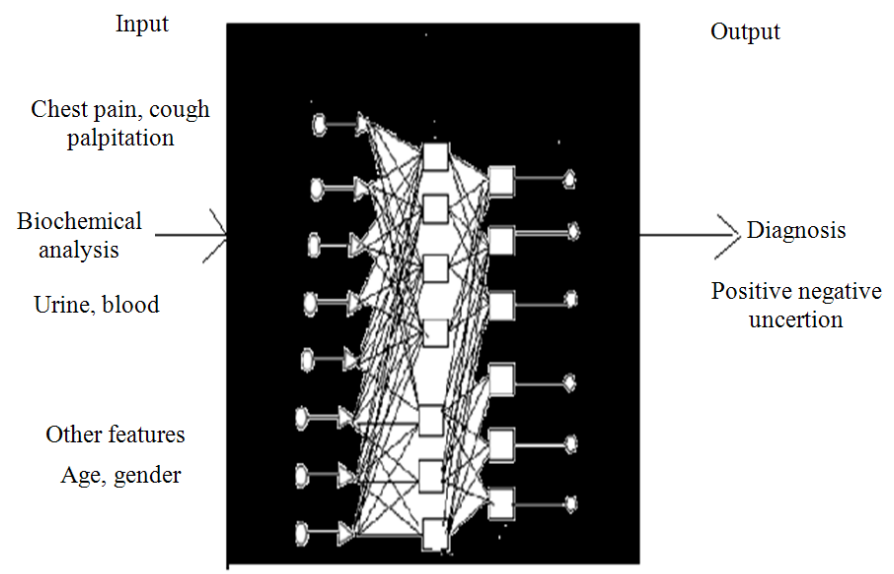

Fig. 3. ANNs-based diagnosis using inputs and outputs

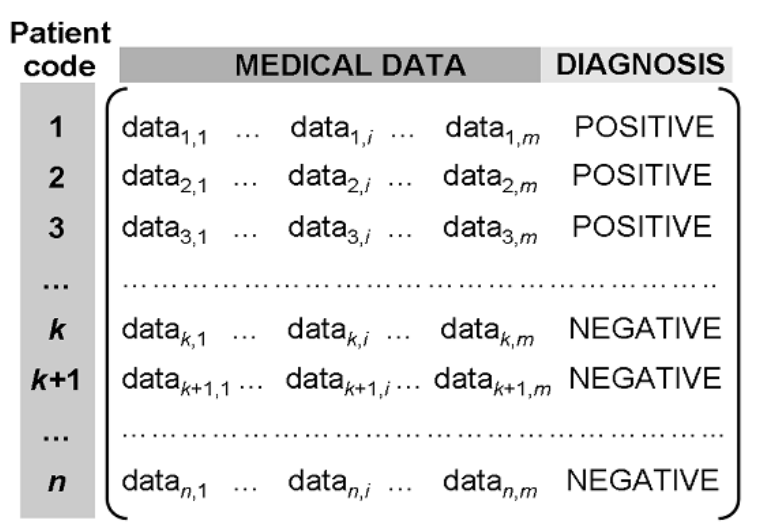

Fig. 4. Training database structure. Different patient labeled in each row with a numerical code. The element datak, i refers to the i-th medical data (symptom, laboratory data) of the k-th patient

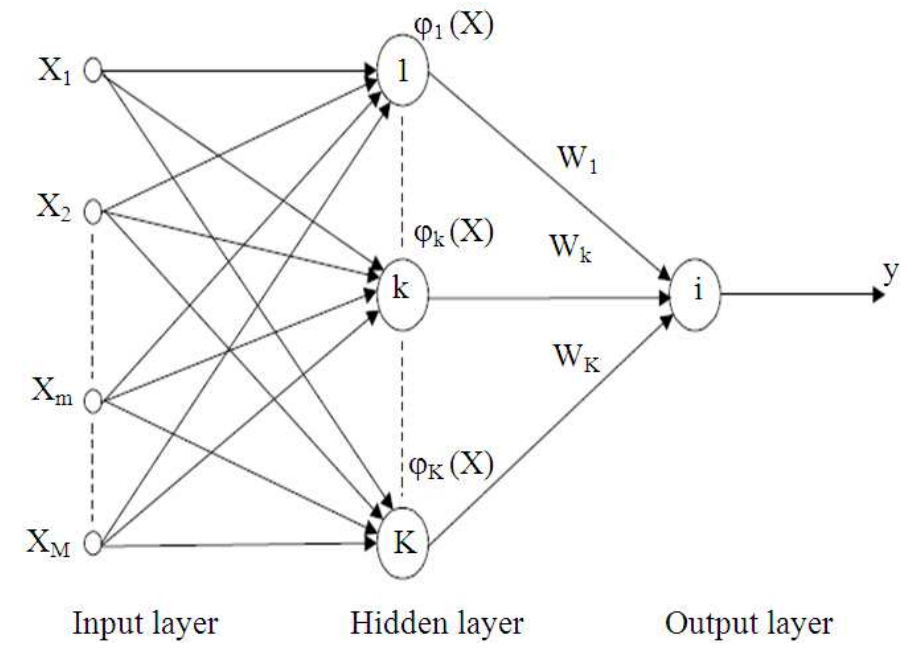

Fig. 5. Generalized regression neural network architecture 
The distance to all the other training samples is bigger. A bigger distance, $D_{j}$, causes the term $\exp \left(-\mathrm{D}_{\mathrm{j}}^{2} / 2 \sigma^{2}\right)$ to become smaller and therefore the contribution of the other training samples to the prediction is relatively small. The term $\mathrm{Y}_{\mathrm{j}}{ }^{*} \exp \left(-\mathrm{D}_{\mathrm{j}}{ }^{2} / 2 \sigma^{2}\right)$ for the $\mathrm{j}^{\text {th }}$ trainng sample is the biggest one and contributes very much to the prediction. The standard deviation or the smoothness parameter, $\sigma$, as it is named in is subject to a search. For a bigger smoothness parameter, the possible representation of the point of evaluation by the training sample is possible for a wider range of $\mathrm{X}$. For a small value of the smoothness parameter the representation is limited to a narrow range of $\mathrm{X}$, respectively.

With (Equation 3) it is possible to:

- Predict behavior of systems based on few training samples

- $\quad$ Predict smooth multi-dimensional curves

- Interpolate between training samples

In (Fig. 6) a prediction performed by GRNN is shown. The circles represent the data points or training samples used to predict the solid line going through most of these samples. The bell shaped curves are the individual terms of (Equation 3) Each of these curves is one term, $Y_{j}{ }^{*} \exp \left(-D_{j}^{2} / 2 \sigma^{2}\right) / \sum_{i=1}{ }^{n} \exp \left(-D_{j}{ }^{2} / 2 \sigma^{2}\right)$ of the whole equation (Equation 3) used in GRNN for the prediction. These terms are normalized normal distributions. Summing up the values of the individual terms at each position yields the value of the prediction, the solid line going through most of the data points. The smoothness parameter was arbitrarily chosen to $\sigma=0.1$.

\section{EXPERIMENTAL RESULT}

The diagnosis of the disease for a new patient to be performed on basis of the makers is facilitated by the primary phase. At the outset, the system intends to diagnosis the kind of hepatitis B: or others: It is necessary to specify the values of the three markers namely Hepatitis B surface Antigen (AgHBs), anti VHC and anti-VHD. Table 4 shows a few sample results. Figure 7 shows the status of the Hepatitis B once the patient is diagnosed positive for hepatitis B.

Table 4. Shows a few sample results

\begin{tabular}{lllllllll}
\hline HBsAg & Anti VHD & HbeAg & Anti VHC & HBc Anti & HBs Anti & $\begin{array}{l}\text { HBc } \\
\text { Anti IgM }\end{array}$ & Result & Status of Patient \\
\hline 0 & 0 & 0 & 0 & 0 & 0 & 0 & 0 & $\begin{array}{l}\text { Susceptible, } \\
\text { Not infected, not immune } \\
\text { Immune due to }\end{array}$ \\
0 & 0 & 0 & 0 & 0 & 1 & 1 & 0 & $\begin{array}{l}\text { Hepatitis B Vaccination } \\
\text { Chronically Infected }\end{array}$ \\
1 & 0 & 0 & 0 & 1 & 0 & 0 & 1 & $\begin{array}{l}\text { Chronically Infected } \\
\text { Acutely infected }\end{array}$ \\
1 & 0 & 0 & 0 & 1 & 0 & 0 & 1 & 1 \\
\hline
\end{tabular}

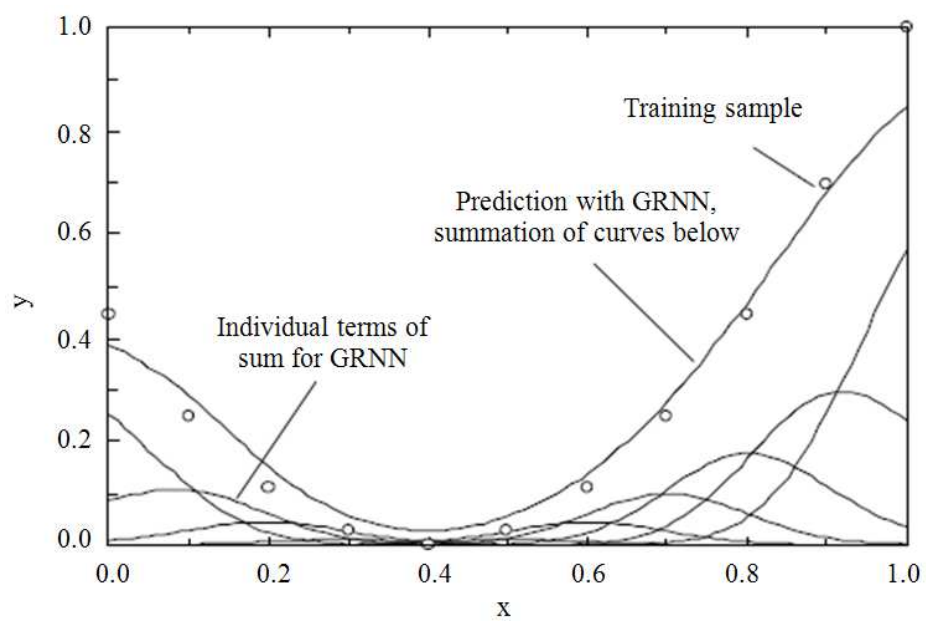

Fig. 6. GRNN with individual terms 


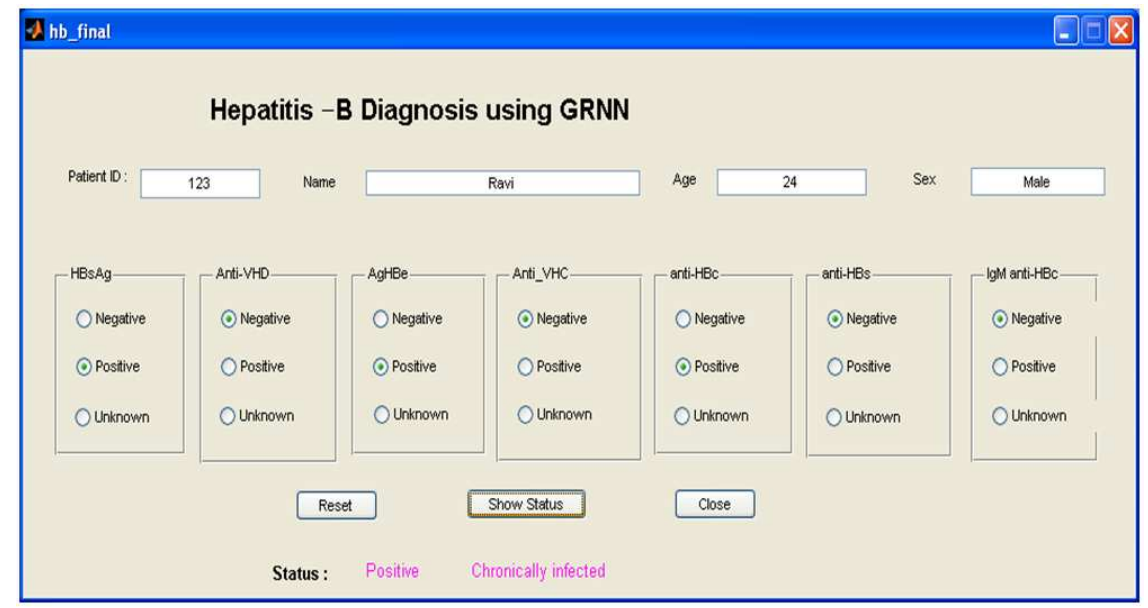

Fig. 7. Hepatitis B diagnosis generalized regression neural network

\section{CONCLUSION}

Hepatitis B is a potentially life-threatening liver infection caused by the hepatitis B virus. The virus interferes with the function of the liver while replicating in hepatocytes. It is a major global health problem and the most serious type of viral hepatitis. Chronic liver disease is caused by viral hepatitis and putting people at high risk of death from cirrhosis of the liver and liver cancer. Hepatitis $\mathrm{B}$ virus is transmitted between people by direct blood-toblood contact or semen and vaginal fluid of an infected person. Modes of transmission are the same as those for the Human Immunodeficiency Virus (HIV), but the hepatitis B virus is 50 to 100 times more infectious. Unlike HIV, the hepatitis B virus can survive outside the body for at least seven days. During this time, the virus can still cause infection if it enters the body of a person who is not protected by the vaccine. The advantages of GRNN based Hepatitis B diagnosis relative to other nonlinear regression techniques are as follows. (a) The network "learns" in one pass through the data and can generalize from examples as soon as they are stored. (b) The estimate converges to the conditional mean regression surfaces as more and more examples are observed; yet, as indicated in the examples, it forms very reasonable regression surfaces based on only a few samples. (c) The estimate is bounded by the minimum and maximum of the observations. (d) The estimate cannot converge to poor solutions corresponding to local minima of the error criterion (as sometimes happens with iterative techniques). (e) A software simulation is easy to write and use. (f) The network can provide a mapping from one set of sample points to another. If the mapping is one to one, an inverse mapping can easily be generated from the same sample points. Generalized regression
N/N (GRNN) will be the best suitable Neural Network for Hepatitis B diagnosis which will help in reducing extra time consumption in treatment. Even if there is any number of missing parameters in blood test, the diagnosis will be done by artificial intelligence using generalized regression neural networks.

\section{REFERENCES}

Aleksander, I. and H. Morton, 1995. An Introduction to Neural Computing. 1st Edn., Internat. Thomson Computer Press, London, ISBN-10: 1850321671, pp: 284.

Brause, R.W., 2001. Medical Analysis and Diagnosis by Neural Networks. In: Medical Data Analysis, Crespo, J., V. Maojo and F. Martin (Eds.), Springer, Berlin, ISBN-10: 3540427341, pp: 1-13.

Cheung, 2003. Machine learning techniques for medical analysis. B.Sc. Thesis, University of Queenland.

MNI, 1996. Hepatitis B (cont.). MedicineNet, Inc.

Ozyilmaz, L. and T. Yildirim, 2003. Artificial neural networks for diagnosis of hepatitis disease. Proceedings of the International Joint Conference on Neural Networks, Jul. 20-24, IEEE Xplore Press, pp: 586-589. DOI: 10.1109/IJCNN.2003.1223422

Stuyver, L., S. De Gendt, C.V. Geyt, F. Zoulim and M. Fried et al., 2000. A new genotype of hepatitis B virus: Complete genome and phylogenetic relatedness. J. Gen. Virol., 81: 67-74. PMID: 10640543

Zupan, J. and J. Gasteiger, 1999. Neural networks in Chemistry and Drug Design. 1st Edn., Wiley VCH, Weinheim, Weinheim, ISBN-10: 3527297782, pp: 380. 